\title{
Cinaciguat Prevents Postnatal Closure of Ductus Arteriosus by Vasodilation and Anti-remodeling in Neonatal Rats
}

\author{
Yu-Chi Hung 1,2, Yi-Ching Liü, Bin-Nan Wu ${ }^{1,4,5}$, Jwu-Lai Yeh ${ }^{1,4,5,6 *}$ and Jong-Hau Hsu ${ }^{3,7 *}$ \\ ' Graduate Institute of Medicine, College of Medicine, Kaohsiung Medical University, Kaohsiung, Taiwan, ${ }^{2}$ Department of \\ Pediatrics, St. Joseph Hospital, Kaohsiung, Taiwan, ${ }^{3}$ Department of Pediatrics, Kaohsiung Medical University Hospital, \\ Kaohsiung Medical University, Kaohsiung, Taiwan, ${ }^{4}$ Department of Pharmacology, College of Medicine, Kaohsiung Medical \\ University, Kaohsiung, Taiwan, ${ }^{5}$ Department of Medical Research, Kaohsiung Medical University, Kaohsiung, Taiwan, \\ ${ }^{6}$ Department of Marine Biotechnology and Resources, National Sun Yat-sen University, Kaohsiung, Taiwan, ${ }^{7}$ Department \\ of Pediatrics, School of Medicine, College of Medicine, Kaohsiung Medical University, Kaohsiung, Taiwan
}

OPEN ACCESS

Edited by:

Agustín Guerrero-Hernández, Centro de Investigaciones y Estudios Avanzados, Instituto Politécnico Nacional de México (CINVESTAV),

Mexico

Reviewed by:

Eliana Hiromi Akamine,

University of São Paulo, Brazil

Luciana Venturini Rossoni,

University of São Paulo, Brazil

*Correspondence:

Jwu-Lai Yeh

jwulai@kmu.edu.tw

Jong-Hau Hsu

jhh936@yahoo.com.tw

Specialty section:

This article was submitted to

Vascular Physiology,

a section of the journal

Frontiers in Physiology

Received: 02 February 2021 Accepted: 06 July 2021

Published: 29 July 2021

Citation:

Hung Y-C, Liu Y-C, Wu B-N, Yeh J-L and Hsu J-H (2021)

Cinaciguat Prevents Postnatal Closure of Ductus Arteriosus by Vasodilation and Anti-remodeling

in Neonatal Rats.

Front. Physiol. 12:661171. doi: 10.3389/fphys.2021.661171
Closure of the ductus arteriosus (DA) involves vasoconstriction and vascular remodeling Cinaciguat, a soluble guanylyl cyclase (sGC) activator, was reported with vasodilatory and anti-remodeling effects on pulmonary hypertensive vessels. However, its effects on DA are not understood. Therefore, we investigated whether cinaciguat regulated DA patency and examined its underlying mechanisms. In vivo, we found that cinaciguat (10 mg/kg, i.p. at birth) prevented DA closure at $2 \mathrm{~h}$ after birth with luminal patency and attenuated intimal thickening. These anti-remodeling effects were associated with enhanced expression of cyclic guanosine monophosphate (cGMP) in DA. Ex vivo, cinaciguat dilated oxygen-induced DA constriction dose-dependently. Such vasodilatory effect was blunted by KT-5823, a PKG inhibitor. In DA smooth muscle cells (DASMCs), we further showed that cinaciguat inhibited angiotensin II (Ang II)-induced proliferation and migration of DASMCs. In addition, cinaciguat inhibited Ang Il-induced mitochondrial reactive oxygen species (ROS) production. Finally, Ang II-activated MAPKs and Akt were also inhibited by cinaciguat. In conclusion, cinaciguat prevents postnatal DA closure by vasodilation and anti-remodeling through the cGMP/PKG pathway. The mechanisms underlying anti-remodeling effects include anti-proliferation and anti-migration, with attenuation of mitochondrial ROS production, MAPKs, and Akt signaling. Thus, this study implicates that SGC activation may be a promising novel strategy to regulate DA patency.

Keywords: ductus arteriosus, soluble guanylyl cyclase, remodeling, vasoconstriction, vascular smooth muscle

\section{INTRODUCTION}

Ductus arteriosus (DA) is a vital vessel connecting the pulmonary arteries and aorta during fetal period and mostly will be closed soon after birth. Patent DA is the major cause of co-morbidities in preterm newborn and, on the other hand, is life-saving in newborns with ductus-dependent congenital heart disease (Hamrick and Hansmann, 2010). Thus, the mechanisms of regulating DA patency are important issues in the field of vascular research. 
Ductus arteriosus closure involves two phases: functional and anatomic closure (Bokenkamp et al., 2010). DA constriction causes functional closure within hours after birth, and subsequent vascular remodeling results in permanent anatomic closure. After birth, increased oxygen tension and declined prostaglandin $\mathrm{E}_{2}\left(\mathrm{PGE}_{2}\right)$ are two major factors for DA constriction. Subsequent DA remodeling is composed of complex histological changes, such as extracellular matrix deposition, and intimal thickening caused by proliferation and migration of smooth muscle cells (SMCs).

Current medications for regulating DA patency is mainly mediated by the PG pathway, with cyclic adenosine monophosphate (cAMP) as the second messenger (Yokoyama et al., 2013). For medical closure of the PDA in preterm newborns with heart failure, PG synthetase inhibitors such as indomethacin or ibuprofen are widely used. For treating newborns with ductus-dependent congenital heart defects (CHDs), $\mathrm{PGE}_{1}$ is the only choice to keep the DA patent for maintaining pulmonary circulation. However, both PG synthetase inhibitors and $\mathrm{PGE}_{1}$ have several significant adverse events (Lewis et al., 1981; Oncel and Erdeve, 2015). Therefore, developing a novel pharmacological target is essential for newborns with PDAs and ductus-dependent CHDs.

Cinaciguat, a soluble guanylyl cyclase (sGC) activator, could activate heme-deficient sGC to increase intracellular cyclic guanosine monophosphate (cGMP) for further biological effects and is currently used in patients with pulmonary hypertension (Evgenov et al., 2006). Previous studies have shown that cinaciguat declined pulmonary pressure and resistance because of vasodilation (Evgenov et al., 2007; Chester et al., 2011) and inhibited vascular remodeling through anti-proliferation and anti-migration of vascular smooth muscle cells (VSMCs) (Dumitrascu et al., 2006; Hirschberg et al., 2013). However, there is no study investigating whether cinaciguat could prevent DA closure.

In this study, we hypothesize that cinaciguat could prevent postnatal DA closure through vasodilatory and anti-remodeling effects on neonatal rats. We also explored the molecular mechanisms underlying these effects on DA smooth muscle cells (DASMCs) treated with angiotensin II (Ang II), a mediator implicated in DA closure (Crockett et al., 2019; Hamrick et al., 2020).

\section{MATERIALS AND METHODS}

\section{Animals}

Timed pregnant Wistar rats were purchased from BioLASCO Taiwan Co., Ltd. (Taipei, Taiwan). The DA was obtained from a newborn rat on the 21st gestational day (full term). This study was approved by the Animal Care and Use Committee of the Kaohsiung Medical University. The Institutional Animal Care and Use Committee (IACUC) number is 102094. The animals were cared for in compliance with the guiding principles of the National Institutes of Health of the United States.

\section{Reagents}

Cinaciguat was purchased from MedChemExpress (Princeton, NJ, United States). Ang II, $\beta$-actin and $\alpha$-smooth muscle actin antibodies, and MTT were obtained from Sigma-Aldrich Inc. (St. Louis, MO, United States). MitoSOX was purchased from Molecular Probes (Eugene, OR, United States). Antibodies against ERK1/2, Akt, and antibodies against phosphorylated ERK, JNK, Akt were obtained from Cell Signaling Technology (Beverly, MA, United States). Antibodies against JNK was obtained from R\&D Systems (Minneapolis, MN, United States). Antibodies against p38 were purchased from Santa Cruz Biotechnology, Inc. (Dallas, TX, United States). Antibodies against phosphorylated p38 were obtained from Abcam (Cambridge, United Kingdom). Dulbecco's Modified Eagle Medium (DMEM), FBS, penicillin, streptomycin, and all other tissue culture reagents were purchased from Gibco BRL Life Technologies (Grand Island, NY, United States).

\section{Morphological Analysis of DA Closure}

To determine whether cinaciguat could prevent DA closure in vivo, newborn rats were injected intraperitoneally with cinaciguat $(10 \mathrm{mg} / \mathrm{kg})$ in the cinaciguat group or saline $(10 \mathrm{ml} / \mathrm{kg})$ in the control group immediately following spontaneous delivery by the pregnant rat on the 21 st gestational day (full term). After injection, the neonates were incubated in room air at $33^{\circ} \mathrm{C}$. Half of the rats in each group were sacrificed at two time points, 0 or $2 \mathrm{~h}$ after injection, by decapitation followed by opening the chest wall. The morphology of the DA and main pulmonary artery (PA) was observed under a dissecting microscope to measure the external diameter of DA and PA. For determining the degree of DA closure, we calculated the ratio of DA/PA.

\section{Histological Analysis of Luminal Patency and Intimal Thickening}

Following morphological analysis, the DA was taken down, and the sectioned segments from the middle portion of the DA were analyzed histochemically. Paraffin-embedded blocks containing these tissues were cut into 3 - $\mu$ m-thick sections and placed on glass slides. We used Orcein stain to reveal the internal elastic lamina, the boundary line between the intima and media layers of the DA, for further determining intimal thickening and medial area of the DA. The intimal thickening and luminal area were estimated by (intimal area)/(medial area) and (luminal area)/(total vessel area), respectively, as previously described (Yeh et al., 2018). The average of at least three sections was used as the value for each tissue.

\section{Immunofluorescence of DA}

Frozen sections of DA were cut into 10 - $\mu \mathrm{m}$-thick sections and placed on glass slides and mounted on appropriately labeled slides. Immunofluorescence staining for the target proteins were performed as described (Liou et al., 2020). The sections were incubated with $10 \%$ paraformaldehyde solution. After washout in PBS plus $0.05 \%$ Triton $\mathrm{X}-100$, the sections were blocked for $30 \mathrm{~min}$ with 5\% BSA and then incubated with rabbit antibodies against cGMP (1:100 dilution; Bioss Inc., ACD, 
Newark, CA, United States) overnight at $4^{\circ} \mathrm{C}$. Then, the sections were incubated with a secondary Goat Anti-Rabbit IgG Antibody $(\mathrm{H}+\mathrm{L})$ FITC conjugate (dilution 1:100; Sigma-Aldrich, St. Louis, MO, United States) at room temperature. The sections were mounted and viewed with a confocal laser-scanning microscope. The average of at least three sections was used as the value for each tissue.

\section{Isometric Tension of the DA Vascular Rings}

To determine whether cinaciguat could inhibit oxygen-induced constriction ex vivo, newborn rats were killed by decapitation to harvest the DA ring immediately after spontaneous delivery by pregnant rats on the 21 st gestational day (full term). The DA was placed in a tissue bath and kept at $37^{\circ} \mathrm{C}$. Two stainless steel wires (40- $\mu \mathrm{m}$ internal diameter) were threaded into the lumen, and the preparation was mounted in a four-channel myograph (Multi-Wire Myograph System 620M, Aarhus, Denmark). One stainless steel wire was connected to a micromanipulator, and the other was connected to a force transducer. All the DA rings were initially stabilized for at least 60 min with a modified KrebsHenseleit solution, which was equilibrated under hypoxia $(0 \%$ $\mathrm{O}_{2}, 5 \% \mathrm{CO}_{2}$, and $95 \% \mathrm{~N}_{2}$ ) and maintained at $37^{\circ} \mathrm{C}$ by a heated water jacket. The isometric tension was continuously monitored using a PowerLab 4/26 system (AD Instruments, Inc., Colorado Springs, CO, United States), as previously described (Yeh et al., 2018). All the vascular rings were stabilized and relaxed, with the resting tension adjusted to $5 \mathrm{mN}$. DA preconstriction was attained by hyperoxia exposure $\left(95 \% \mathrm{O}_{2}\right.$ and $\left.5 \% \mathrm{CO}_{2}\right)$. The vasodilatory effect of cinaciguat $(1-100 \mathrm{nM})$ was assessed at peak $\mathrm{O}_{2}$ constriction by the application of incremental doses at 5min intervals. To determine the role of the PKG pathway, we examined the effects of cinaciguat on $\mathrm{O}_{2}$-induced DA contraction by pretreatment with KT5823 (10 $\mu \mathrm{M})$, a PKG inhibitor, $10 \mathrm{~min}$ before hyperoxia exposure. At the end of the experiments, vasoconstriction of the $\mathrm{DA}$ was induced by $\mathrm{KCl}(50 \mathrm{mM})$ containing Krebs-Henseleit solution to assure vessel reactivity.

\section{Primary Culture of Rat DASMCs}

DA smooth muscle cells in primary culture were all obtained from the DA of newborn Wistar rats as previous described (Yeh et al., 2018). In brief, immediately after spontaneous delivery by the pregnant rat on the 21st gestational day, the newborn rats were killed by decapitation followed by taking down the DA. The outer sphere of the DA was peeled, and the intimal layer of DA was shaved lightly two to three times to remove endothelial cells. DAs were then plated onto Petri dishes (Corning Inc., Corning, NY, United States) and cultured in DMEM supplemented with $10 \% \mathrm{FBS}, 100 \mathrm{U} / \mathrm{ml}$ of penicillin, and $100 \mu \mathrm{g} / \mathrm{ml}$ streptomycin, maintained in a humidified incubator $\left(37^{\circ} \mathrm{C}, 5 \% \mathrm{CO}_{2}\right.$, and 95\% air). When the cultures reached confluence, cells were subcultured using $0.5 \%$ trypsin. The purity of the DASMCs was confirmed under a confocal microscope by immunofluorescence staining for $\alpha$-smooth muscle actin (>95\% of cells stained positive), with a typical "hill and valley" appearance and lack of staining for vimentin, as previously described (Wu et al., 2016).
Serum starvation before the experiments was achieved by $1 \%$ FBS for $48 \mathrm{~h}$ to synchronize cells into quiescence.

\section{Cell Proliferation Assay}

To assess DASMC proliferation, an MTT assay was first performed as previously described (Hsu et al., 2011). In brief, after serum starvation for $48 \mathrm{~h}$, proliferation of DASMCs was induced by Ang II (10-nM) for $48 \mathrm{~h}$ in DMEM supplemented with $1 \%$ FBS in 96-well plates at a density of $4 \times 10^{4}$ cells per well, with or without pretreatment $(1 \mathrm{~h})$ and co-treatment $(48 \mathrm{~h})$ with cinaciguat $(0.1-10 \mu \mathrm{M})$. After induction for $48 \mathrm{~h}$, MTT $(0.5 \mathrm{mg} / \mathrm{ml})$ was added to the medium for $4 \mathrm{~h}$. The culture medium was then removed, and the cells were dissolved in isopropanol and shaken for $10 \mathrm{~min}$. The amount of MTT formazan was quantified at absorbances of 540 and $630 \mathrm{~nm}$ using an enzyme-linked immunosorbent assay (ELISA) reader (Dynex Technologies, Denkendorf, Germany). In addition, the DNA synthesis of DASMC was also assessed by the bromodeoxyuridine (BrdU) incorporation assay (Roche Applied Science, Mannheim, Germany). The DASMCs were seeded into 96-well plates at a density of $4 \times 10^{4}$ cells per well, and the confluent DASMCs were induced by Ang II (10 nM) with or without cinaciguat $(0-10 \mu \mathrm{M})$ for $24 \mathrm{~h}$ in the same medium containing BrdU $(10 \mu \mathrm{M})$. Cells were harvested for the detection of DNA synthesis by incorporation of BrdU using a cell proliferation ELISA. The DASMCs were measured colorimetrically using a Bio-Rad model $680 \mu \mathrm{L}$ plate reader (Bio-Rad, Hercules, CA, United States) at a wavelength of $450 \mathrm{~nm}$.

\section{Cell Migration Assay}

Migration of DASMCs was assessed on Transwell polyethylene terephthalate cell culture inserts with $8-\mu \mathrm{m}$ pores as previously described (Hsu et al., 2011). After serum starvation for $48 \mathrm{~h}$, DASMCs $\left(2 \times 10^{4}\right.$ cells $)$ were loaded into the upper compartment and incubated for $48 \mathrm{~h}$ at $37^{\circ} \mathrm{C}\left(5 \% \mathrm{CO}_{2}\right.$ and $95 \%$ air). Meanwhile, in the lower compartment, Ang II (10 nM) was dissolved in DMEM, with or without pretreatment $(1 \mathrm{~h})$ and co-treatment $(48 \mathrm{~h})$ with cinaciguat $(0.1-10 \mu \mathrm{M})$. After $48 \mathrm{~h}$, the non-migrated cells on the upper membrane surface were removed, and those on the lower surface were fixed in methanol and stained with Giemsa. The number of cells per six high-power fields $(200 \times \mathrm{HPF})$ was counted, and the mean number of cells was used to express migration activity.

\section{Measurement of Mitochondrial ROS Production}

Mitochondrial reactive oxygen species (ROS) production in DASMCs was measured using MitoSOX, a fluorescent probe specific for mitochondrial superoxide. After serum starvation for $48 \mathrm{~h}$, DASMCs $\left(1 \times 10^{5}\right.$ cells $)$ were stimulated by Ang II $(10 \mathrm{nM})$ for $48 \mathrm{~h}$ with or without pretreatment with cinaciguat (0.1-10 $\mu \mathrm{M})$. MitoSOX (5 $\mu \mathrm{M}$, molecular probes) was added for a $10-\mathrm{min}$ incubation period at room temperature and was fixed by $1 \%$ formaldehyde after washing with PBS. Cellular fluorescence was examined under a fluorescence microscope at an excitation/emission of 510/580 $\mathrm{nm}$ following the instructions 
of the manufacturer. Results were expressed as fluorescence intensities in arbitrary units (AU).

\section{Western Blot Analysis}

The protein samples were extracted from the whole cells following methods previously described (Liou et al., 2004). The extracted protein solutions were stored at $-80^{\circ} \mathrm{C}$ until analysis. Equivalent amounts of protein were resolved by SDS-polyacrylamide gel electrophoresis (PAGE) (10-14\%) and transferred to polyvinylidene difluoride membranes. After blocking for $1 \mathrm{~h}$ in $5 \%$ non-fat dry milk and Tris-buffered saline, the membrane was incubated with the desired primary antibody for $2 \mathrm{~h}$. The membrane was then treated with the appropriate horseradish peroxidase-conjugated secondary antibody (Chemicon Inc., Temecula, CA, United States), and the immunoreactive bands were detected by enhanced chemiluminescence (ECL) reagents (PerkinElmer Life Sciences Inc., Waltham, MA, United States). Proteins labeled with specific primary and secondary antibodies were visualized with enhanced chemiluminescence. $\beta$-Actin was probed as a control to ensure equal protein loading.

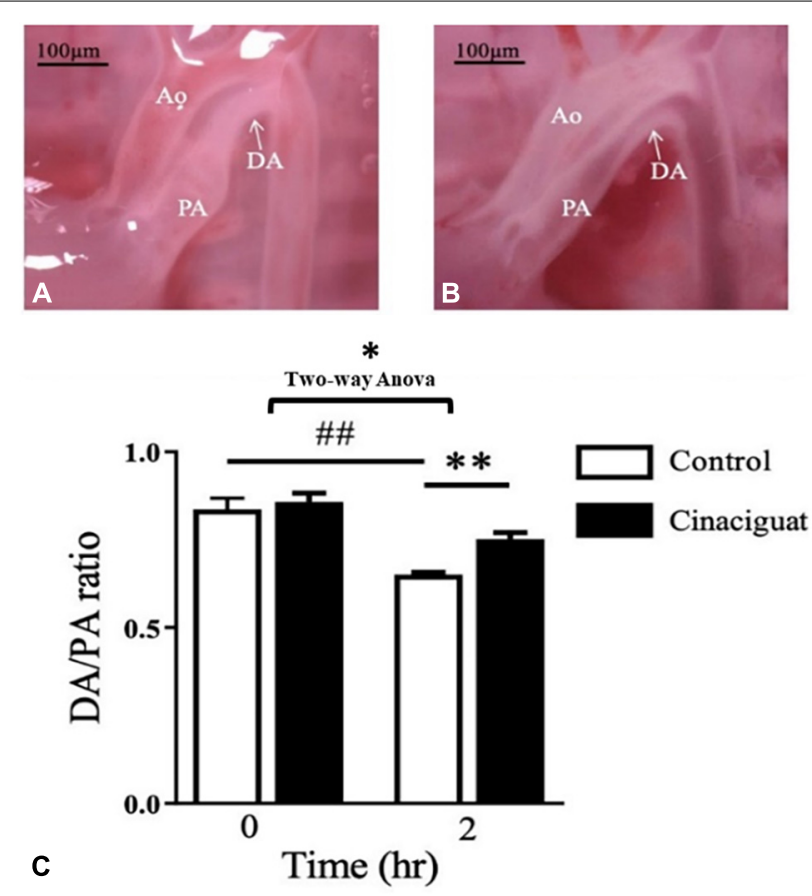

FIGURE 1 | Cinaciguat prevented postnatal ductus arteriosus (DA) closure in neonatal rats. (A) Under dissecting microscope, we found that the DA of control rats closed at $2 \mathrm{~h}$ after birth with luminal obliteration. (B) In cinaciguat-injected rats, cinaciguat (10 mg/kg, i.p. at $0 \mathrm{~h}$ after birth) prevented DA closure with luminal patency at $2 \mathrm{~h}$ after birth. (C) The bar graph showed serial changes in the DA/pulmonary artery $(\mathrm{PA})$ ratio in both groups. In the control group, the ratio declined significantly within $2 \mathrm{~h}$ after birth. Comparing control at $2 \mathrm{~h}$ after birth, cinaciguat group had higher DA/PA ratio. Values represent the mean $\pm \mathrm{SEM} N=6$. Ao, aorta; PA, main pulmonary artery; DA, ductus arteriosus. ${ }^{\star} P<0.05$ by the two-way ANOVA analysis; ${ }^{\# \#}$ and ** $P<0.01$ by the post hoc test; magnification $10 \times$.

\section{Statistical Analysis}

Results are expressed as the mean \pm the standard error (SEM). In the in vivo study, the statistical interactions between DA diameter, luminal patency, intimal thickening, and cinaciguat treatment were obtained by two-way ANOVA or unpaired $t$-test. In the DA tension study, repeated two-way ANOVA was performed to compare the group and time effect. In the in vitro experiments, statistical differences were determined by the one-way ANOVA analysis with Bonferroni's post hoc test. The KolmogorovSmirnov test was performed to examine the normality. A $P<0.05$ was considered statistically significant.

\section{RESULTS}

\section{Cinaciguat Prevented DA Closure at $2 \mathrm{~h}$ After Birth}

To examine the effect of cinaciguat on neonatal rat, we injected cinaciguat into the newborn rats intraperitoneally at $0 \mathrm{~h}$ after birth and then examined the DA condition at $2 \mathrm{~h}$ after birth for comparison with control rats. We used the external diameter ratio of DA/PA to represent the degree of DA closure. In the control rats, the rats at $2 \mathrm{~h}$ after birth had luminal obliteration (Figure 1A) and lower DA/PA ratio compared with those at $0 \mathrm{~h}$ after birth $(65.1 \pm 0.6$ vs. $83.7 \pm 3.1 \%, \boldsymbol{P}<0.01)$ (Figure 1C). At $2 \mathrm{~h}$ after birth, the cinaciguat-injected rats had more patent DA (Figure 1B) and higher DA/PA ratio compared with control rats at $2 \mathrm{~h}$ after birth $(75.2 \pm 1.9$ vs. $65.1 \pm 0.6 \%$, $\boldsymbol{P}<0.01$ ) (Figure 1C).

\section{Cinaciguat Attenuated Intimal Thickening and Maintained Luminal Patency of the DA at $\mathbf{2} \mathrm{h}$ After Birth}

We investigated the histological change in the DA and analyzed the effect of cinaciguat on intimal thickening and luminal area. In the control rats at $2 \mathrm{~h}$ after birth, we found that the lumen of the DA was obstructed with intimal thickening (Figure 2A). In the cinaciguated-injected rats at $2 \mathrm{~h}$ after birth, the lumen of the DA remained patent with minimal intimal thickening (Figure 2B). Quantitatively, we found that cinaciguat attenuated intimal formation as evidenced by ratio of intimal area/medial area $(12.4 \pm 1.2$ vs. $24.4 \pm 1 \%, P<0.01)$ (Figure $2 \mathrm{C}$ ) and maintained luminal patency (ratio of luminal area/total vessel area) $(29.3 \pm 2.6$ vs. $12.4 \pm 0.6 \%, P<0.01)$ (Figure 2D).

\section{Cinaciguat Preserved Expression of cGMP While Maintaining DA Patency}

The cGMP (cyclic guanosine $3^{\prime}, 5^{\prime}$-monophosphate) is an important second messenger in the NO-sGC pathway. It has been previously found that, after birth, there is an oxygen-induced decrease in intracellular cGMP levels in DA (Crockett et al., 2019). To evaluate the cGMP expression in rat DA tissue, we performed immunofluorescence analysis and image quantitation of cGMP in DA sections. At $2 \mathrm{~h}$ after birth, we found that there was a significant decrease in cGMP expression in the control group, but not in the cinaciguat-treated neonatal rats (Figure 3), 

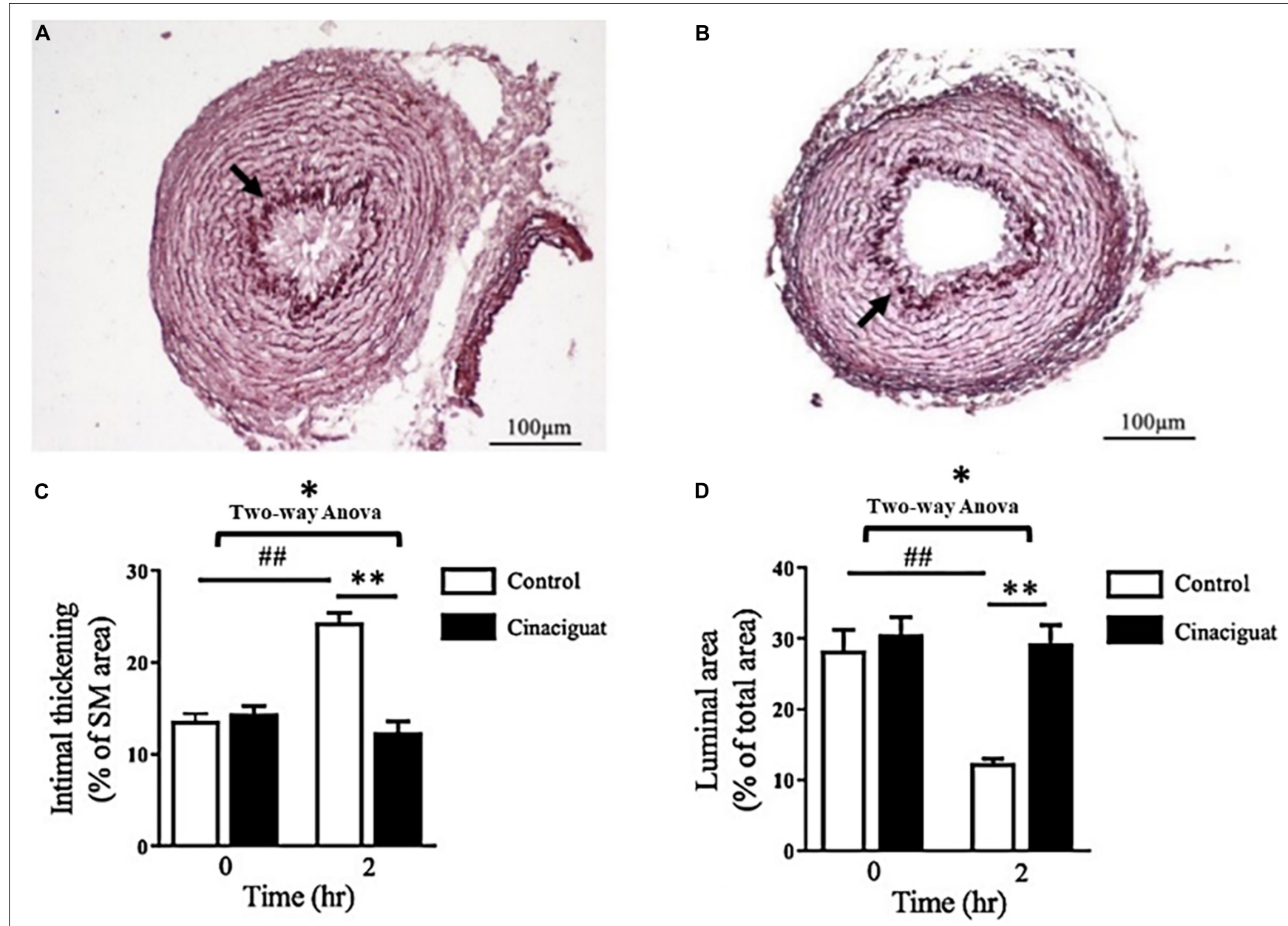

FIGURE 2 | Cinaciguat attenuated intimal thickening and maintained luminal patency of the ductus arteriosus (DA) in neonatal rats. (A,B) Histochemical analysis of DA section showed less intimal thickening and more luminal area in the (B) cinaciguat group compared with the (A) control group at $2 \mathrm{~h}$ after birth. Arrows indicate the internal elastic lamina separating the intimal layer from the medial layer. (C,D) The bar graphs show serial changes in the (C) intimal thickening and (D) luminal area in both groups. (C) In the control group, the intimal thickening (ratio of intima area/media area) increased significantly at $2 \mathrm{~h}$ compared with $0 \mathrm{~h}$, while cinaciguat attenuated such increment at $2 \mathrm{~h}$. (D) In the control group, the luminal patency (ratio of luminal area/total vessel area) was attenuated significantly at $2 \mathrm{~h}$ compared with $0 \mathrm{~h}$, while cinaciguat preserved luminal patency at $2 \mathrm{~h}$. SM, smooth muscle. Values represent the mean $\pm \mathrm{SEM}$; $N=6$. ${ }^{*} P<0.05$ by the two-way ANOVA analysis; ${ }^{\# \#}$ and ${ }^{* \star} P<0.01$ by the post hoc test; magnification $200 \times$.

indicating that cinaciguat prevented DA closure with associated up-expression of cGMP.

\section{Cinaciguat Dilated Oxygen-Induced DA Constriction}

During DA closure, vasoconstriction is an important mechanism besides intimal thickening. Given that oxygen is the main factor for DA constriction, we explored the effect of cinaciguat on the DA ring with an oxygen-rich solution. Cinaciguat declined the vascular tone of oxygen-constricted DA in a dose-dependent manner (Figure 4A). The vascular tone enhanced by oxygen was significantly attenuated by cinaciguat at concentrations of 10 and $100 \mathrm{nM}(66.2 \pm 3.3$ and $90.9 \pm 2.6 \%$, respectively, ANOVA $P<0.01)$. In order to identify the underlying vasodilatory mechanism of cinaciguat, we further pretreated the DA ring with a PKG inhibitor (KT-5823, $10 \mu \mathrm{M}$ ) (Figure 4B) and found that
PKG inhibition attenuated the vasodilatory effects of cinaciguat by 32 and $20 \%$ at concentrations of 10 and $100 \mathrm{nM}$, respectively (both $P<0.01$ ) (Figure 4C).

\section{Cinaciguat Inhibited Ang II-Induced DASMC Proliferation and Migration}

DA smooth muscle cell proliferation and migration constitute intimal thickening for DA closure. Histologically, we found that cinaciguat attenuated intimal thickening with antiremodeling effect, then we investigated underlying cellular and molecular mechanisms via in vitro studies using DASMCs. To determine whether cinaciguat attenuates Ang II-induced DASMC proliferation, we first examined the viability of DASMCs by the MTT assay. The results showed that cinaciguat attenuated Ang II-induced proliferation of DASMCs in a concentrationdependent manner (Figure 5A). In addition to the MTT assay, 

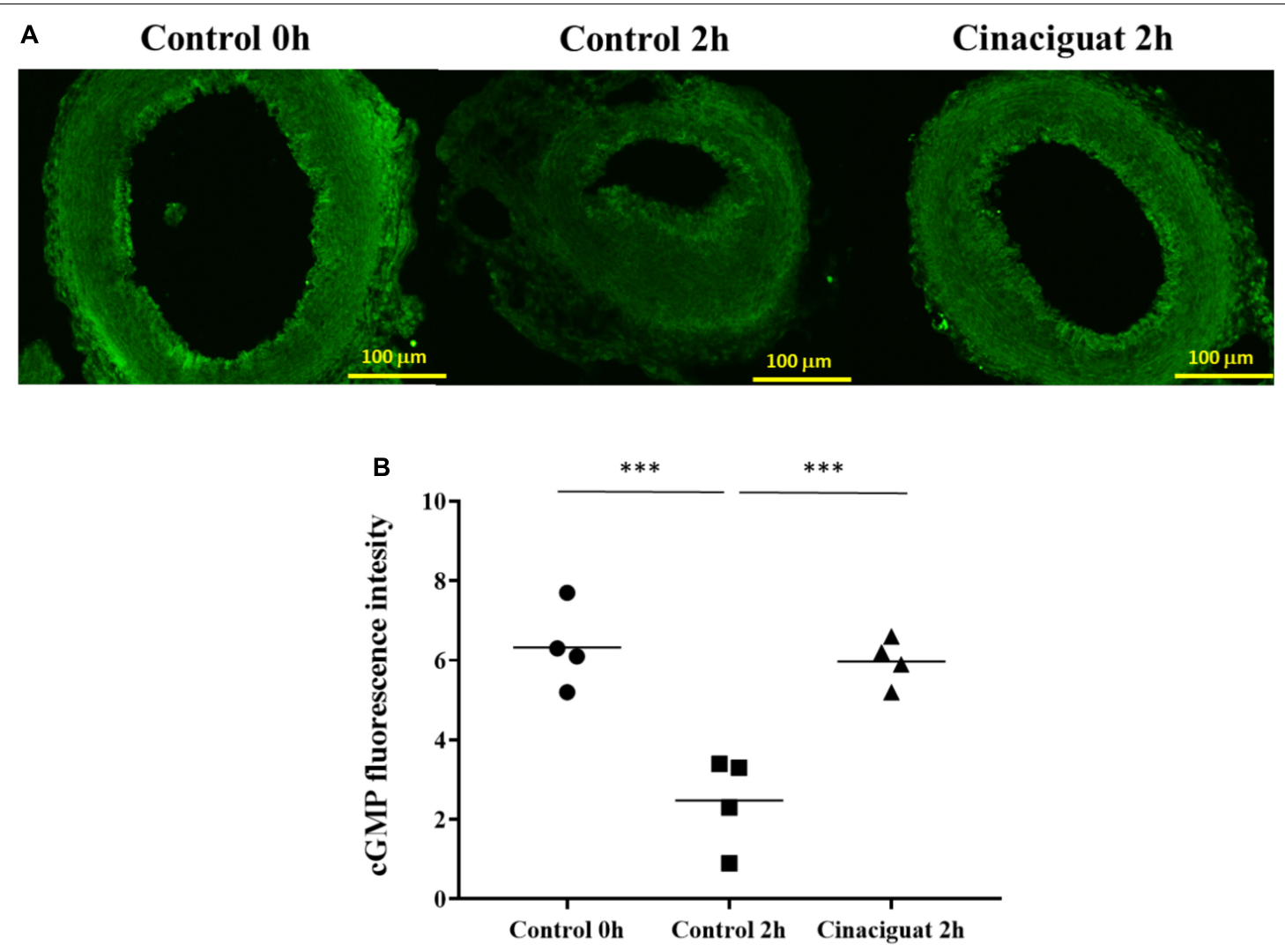

FIGURE 3 | Cinaciguat prevented ductus arteriosus (DA) closure and intimal thickening with associated preservation of cGMP in neonatal rats. (A) Representative immunofluorescence images of cGMP. (B) cGMP expression levels in DA in all groups. Values are presented by the dot plot with the mean. $N=4$. ANOVA $P<0.05$; ${ }^{\star \star *} P<0.001$; magnification $200 \times$.

we performed quantitative colorimetric assay for DNA synthesis based on BrdU incorporation, to examine the antiproliferative effects of cinaciguat. In parallel, we found that cinaciguat attenuated Ang II-induced DNA synthesis of DASMCs dosedependently (Figure 5B). Furthermore, we determined the effects of cinaciguat on DASMC migration induced by Ang II (10 nM) with the Boyden chamber assay. We found that Ang II potently stimulated DASMCs but co-treatment of cinaciguat inhibited Ang II-stimulated migration in a concentration-dependent manner (Figure 5C).

\section{Cinaciguat Inhibited Ang II-Induced Mitochondrial ROS Production}

We used DASMCs to study the mitochondrial ROS production, which may have roles in mediating SMC proliferation and migration. We found that cinaciguat inhibited Ang II-induced mitochondrial ROS production dose-dependently (Figure 6).

\section{Cinaciguat Inhibited Ang II-Induced Phosphorylation of ERK 1/2, JNK, and p38}

Next, we examined the effects of cinaciguat on important signaling in cell proliferation and migration, MAPK signaling including ERK 1/2, JNK, and p38. Cinaciguat attenuated Ang II-induced phosphorylation of ERK 1/2, JNK, and p38 in a dose-dependent manner (Figure 7).

\section{Cinaciguat Inhibited Ang II-Induced Phosphorylation of Akt}

We also examined the effect of cinaciguat on Akt signaling, which is important for cell proliferation and migration. Cinaciguat attenuated Ang II-induced phosphorylation of Akt in a dosedependent manner (Figure 8).

\section{DISCUSSION}

In this study, we found for the first time that cinaciguat, a sGC activator, could prevent DA closure in neonatal rats through vasodilatory and anti-remodeling effects. These effects were mediated, at least partly, by the cGMP/PKG pathway. To elucidate the underlying molecular mechanisms, we further demonstrated that in DASMCs cinaciguat conveyed antiproliferative and anti-migratory effects, with attenuation of mitochondrial ROS and down-regulations of MAPK and Akt signaling. The findings indicate that sGC activation may be a novel pathway to be explored and shed some light in expanding 


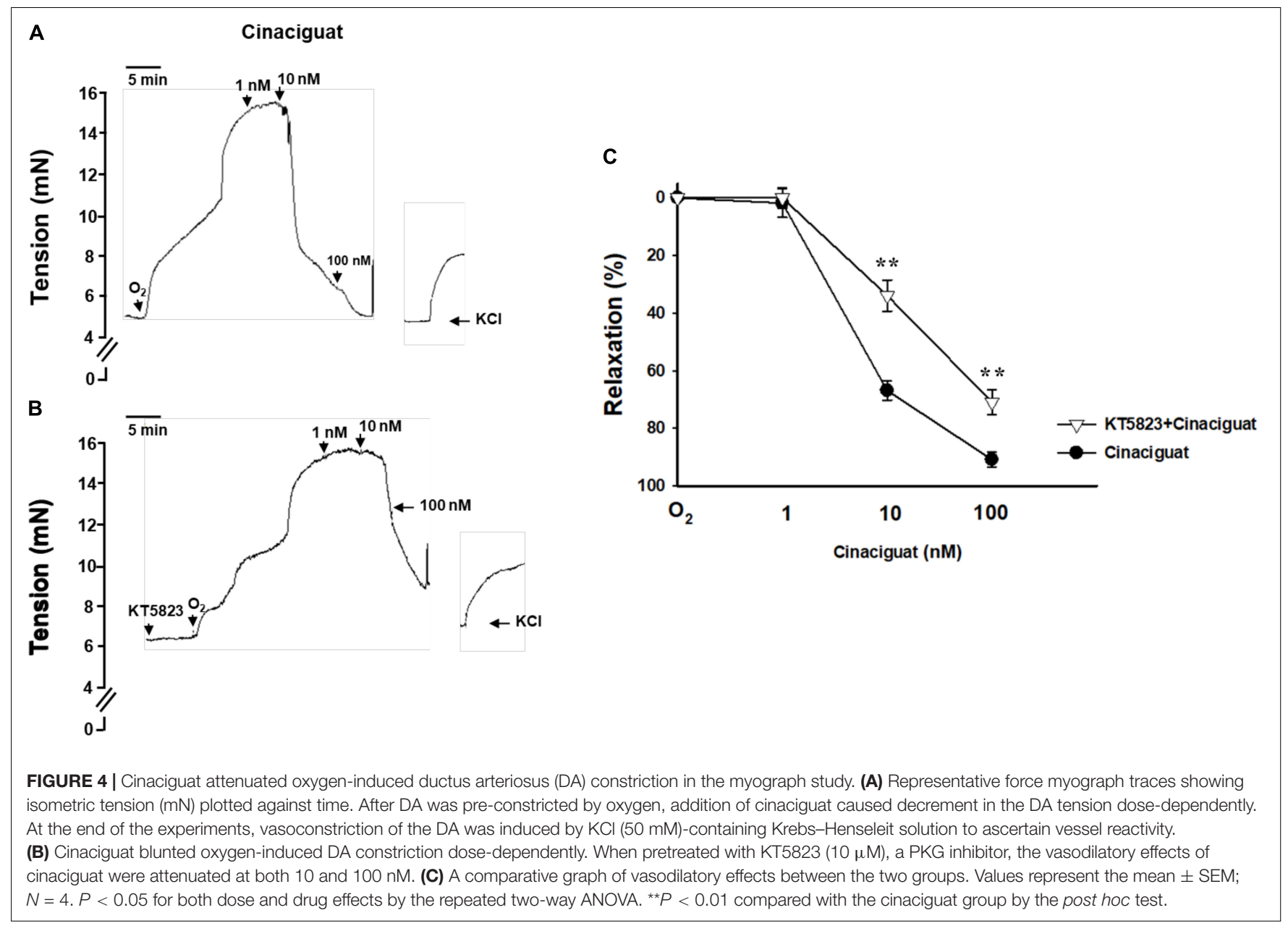

the current pharmacological management of DA patency or DAdependent heart diseases.

Mechanisms underlying the regulation of DA patency are complex and not fully understood (Hung et al., 2018; Lin et al., 2020). Even though the current clinical management mainly targets the prostaglandin pathway, issues such as adverse effects and incomplete responses have been unsolved (Hsu et al., 2010). Therefore, research regarding pathways beyond that of prostaglandin may have clinical implications in DA regulation. Indeed, it has been recently suggested that the NO pathway may have a role in both vasodilatation and anti-remodeling in DA (Hsu et al., 2021). For example, during DA functional closure, the genetic expression of NO synthase (NOS), a key enzyme-producing $\mathrm{NO}$, was found to be significantly altered in the DA (Goyal et al., 2016). In addition, endothelial NOS, the most abundant type of NOS detected in the ductal wall, exerted vasodilatory effect on the DA (Clyman et al., 1998; Ovali, 2020). Beyond the role of NO in functional closure, it has been demonstrated in premature baboons that NO can also attenuate anatomical remodeling of the DA (Seidner et al., 2001). In vascular SMCs, it is known that sGC is the target enzyme of NO. However, tolerance or resistance to NO may limit sGC activity and cGMP production in SMCs, thus hindering vasodilation
(Lakshminrusimha et al., 2016). In addition, it is known that there is a postnatal physiologic decrease in intracellular cGMP levels of the DA (Crockett et al., 2019). Therefore, a strategy of sGC/cGMP activation might be a rational approach in the regulation of DA patency after birth.

Emerging evidence shows that sGC activator cinaciguat can decrease pulmonary pressure through vasodilatory effect and has also inhibited vascular remodeling in studies on pulmonary hypertension. For example, cinaciguat caused pulmonary vasodilation in fetal lambs with chronic intrauterine pulmonary hypertension due to DA ligation (Chester et al., 2011). Similarly, in animals with pulmonary hypertension, cinaciguat attenuated muscularization of pulmonary arteries, suggesting its antiremodeling effect (Dumitrascu et al., 2006). However, the effects of cinaciguat on the DA are still unknown.

In this study, we first demonstrated in vivo that cinaciguat can preserve DA patency in neonatal rats. Since DA patency can be caused by vasodilatation and anti-remodeling, we further investigated these effects on isolated DA rings and by histochemical stains, respectively. To elucidate the effects of vasodilatation, we conducted an ex vivo tension study on DA tissue, and confirmed that cinaciguat dilated the oxygenconstricted DA, and this effect was blunted by the PKG 


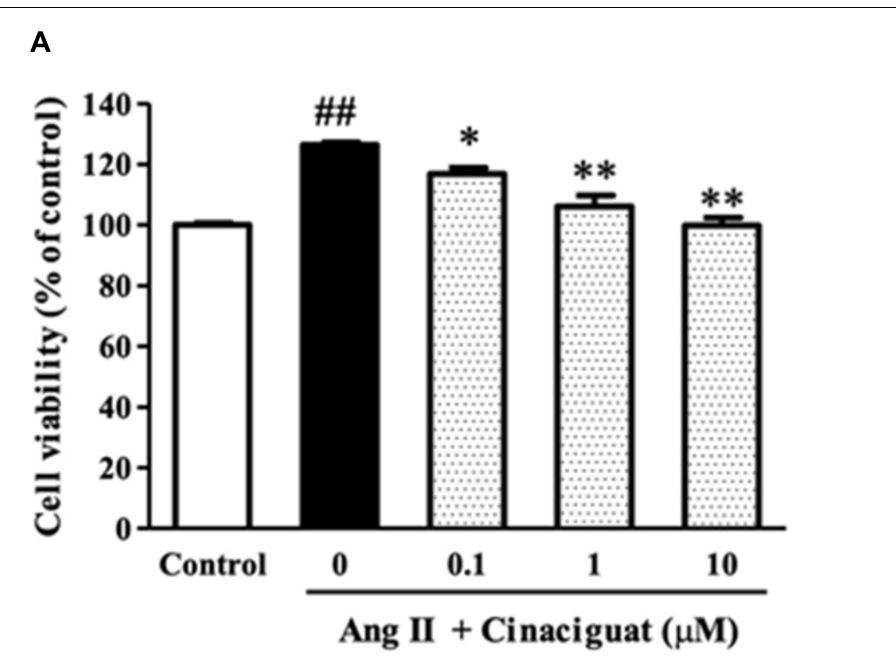

C

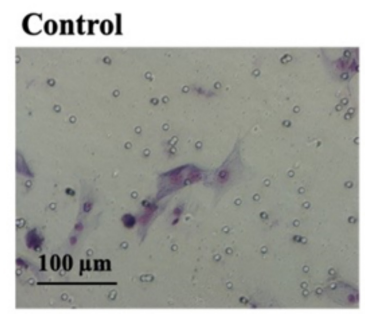

Ang II

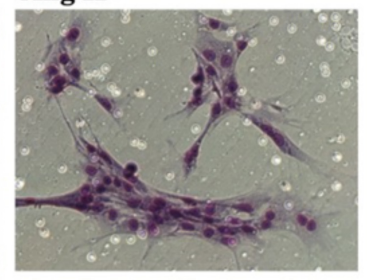

\section{Cinaciguat $1 \mu \mathrm{M}$}

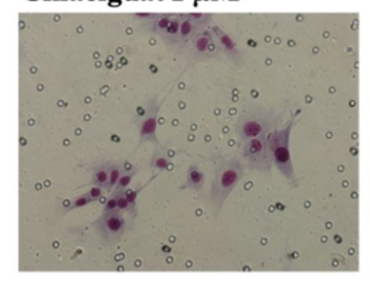

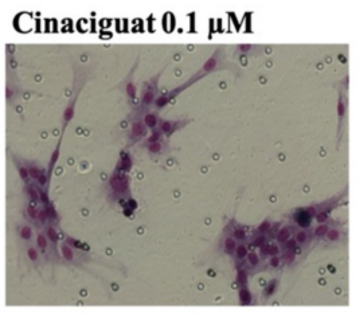

B
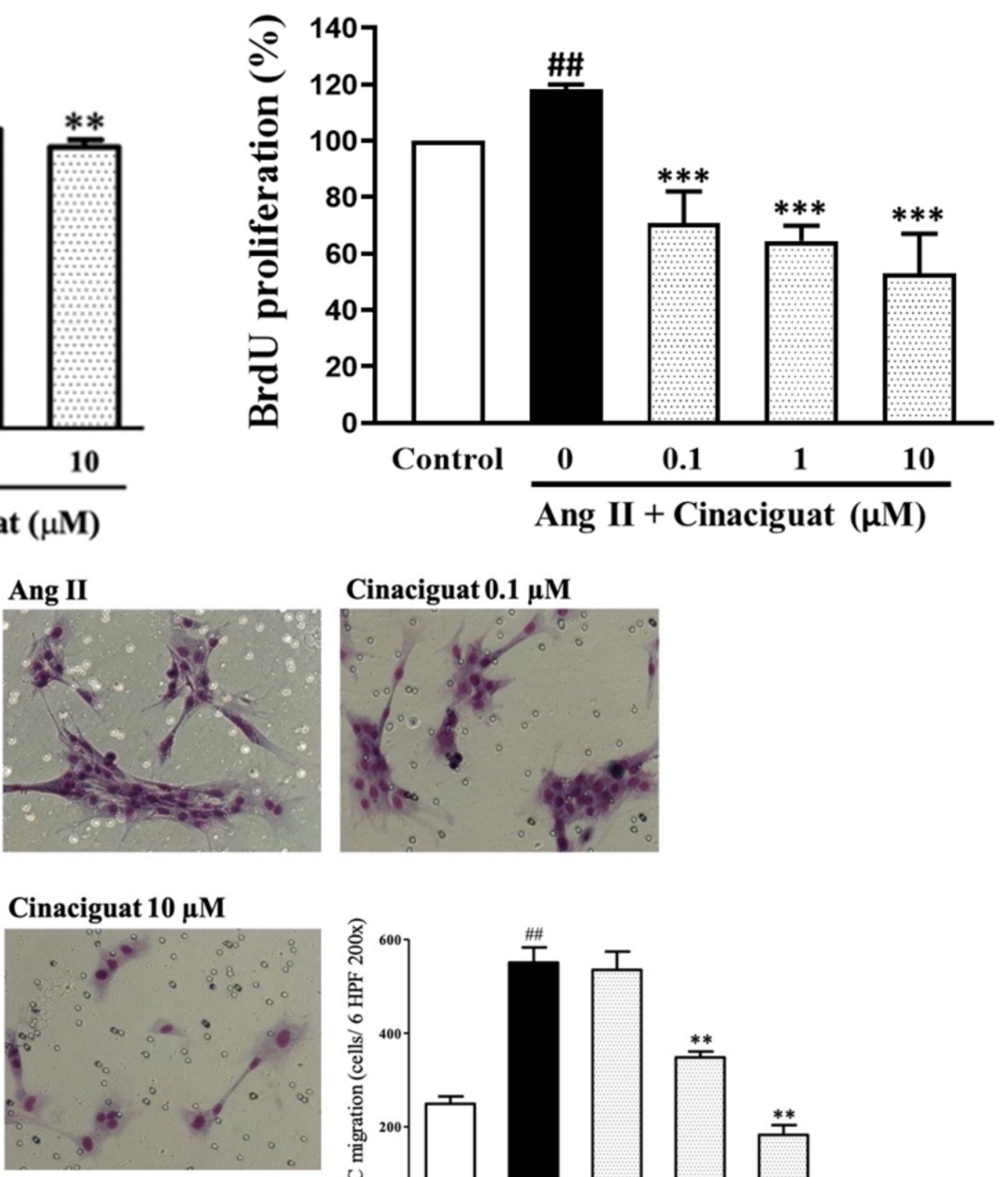

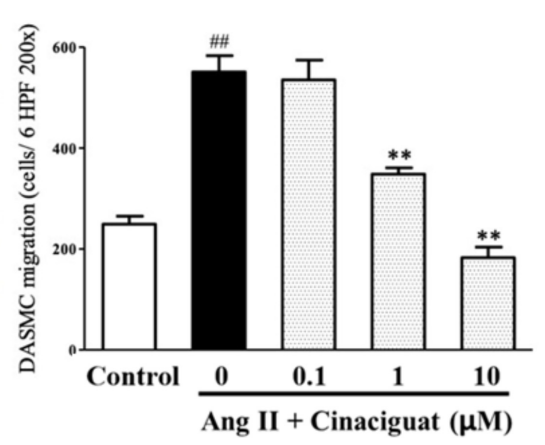

FIGURE 5 | Cinaciguat inhibited Ang II-induced ductus arteriosus smooth muscle cell (DASMC) proliferation and migration. (A) The cell viability was augmented by Ang II after $48 \mathrm{~h}$ of incubation. Cinaciguat significantly inhibited Ang II-induced proliferation in a dose dependent manner. (B) BrdU incorporation of DASMCs was increased after incubation with Ang II for $48 \mathrm{~h}$. However, cinaciguat attenuated Ang II-induced BrdU incorporation in a concentration-dependent manner.

(C) Cinaciguat significantly inhibited Ang II-induced migration in a dose dependent manner. The photomicrograph showed the Ang II-induced migration of DASMCs from upper to lower chamber. The bar graph shows migration activity assessed by the number of cells observed in six high-power fields. Values represent the mean \pm SEM; $N=6$. All one-way ANOVA $P<0.0001 .{ }^{\# \#} P<0.01$ vs. control; ${ }^{*} P<0.05,{ }^{* *} P<0.01$, and ${ }^{* * *} P<0.001$ vs. Ang $\|$ alone (magnification $200 \times$ ).

inhibitor KT5823. In the histochemical stains, we found that cinaciguat could mitigate the intimal thickening and maintained DA luminal patency, which was associated with preserved cGMP expression. Taken together, these findings demonstrate that cinaciguat can prevent DA closure by the cGMP/PKG pathway. Previously, we have shown that BNP can prevent DA closure through the $\mathrm{CGMP} / \mathrm{PKG}$ pathway, which is mediated by particulate GC (pGC) (Yeh et al., 2018). In parallel, this study further shows that activation of the sGC/cGMP pathway can also prevent DA closure by vasodilatation and anti-remodeling.

Proliferation and migration of SMCs are both important factors mediating vascular remodeling. In an animal model of vascular injury, cinaciguat augmented PKG signaling and prevented neointimal formation via decreasing SMC proliferation and migration (Hirschberg et al., 2013). To further determine the mechanisms of the anti-remodeling effect of cinaciguat, we investigated the effects of cinaciguat on DASMC proliferation and migration. We found that cinaciguat inhibited DASMC proliferation and migration induced by Ang II. These results were in line with the previous study showing that through the cGMP pathway, BNP inhibited DA and PA SMC proliferation and migration (Hsu et al., 2014; Yeh et al., 2018).

Reactive oxygen species production is essential in vascular remodeling of pulmonary hypertension and appears to be 


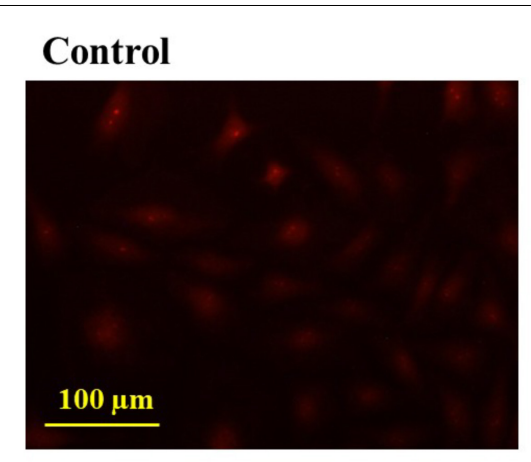

Cinaciguat $1 \mu \mathrm{M}$

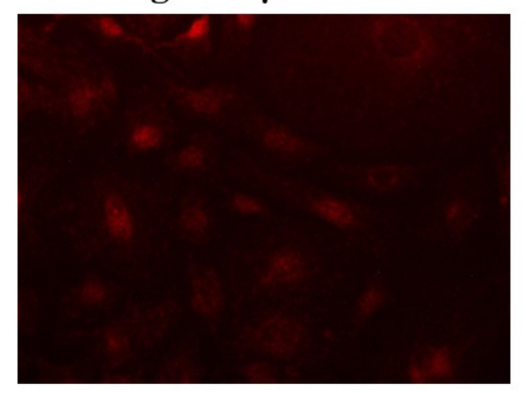

Ang II

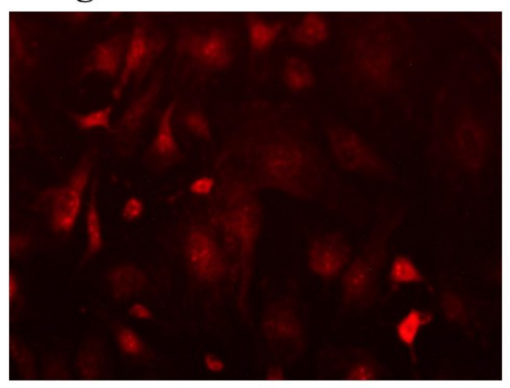

Cinaciguat $10 \mu \mathrm{M}$

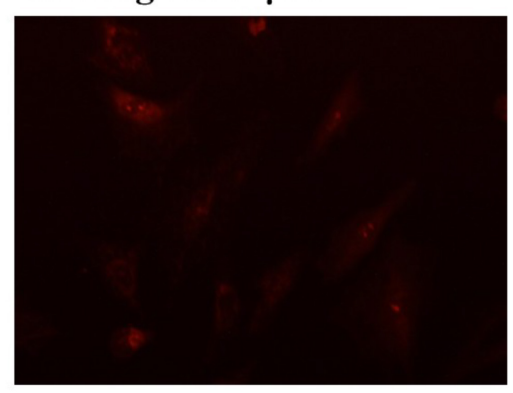

\section{Cinaciguat $0.1 \mu \mathrm{M}$}
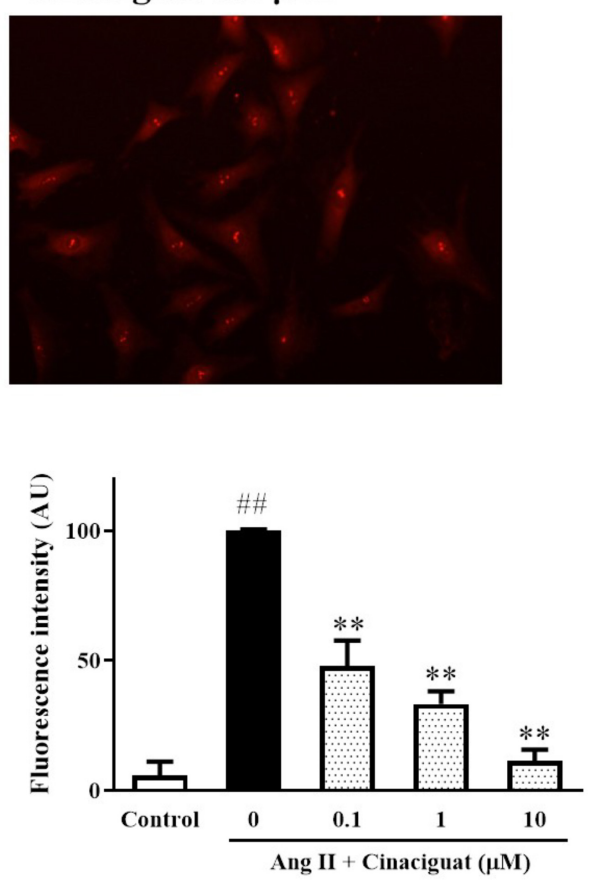

FIGURE 6 | Cinaciguat-inhibited Ang II-induced mitochondrial reactive oxygen species (ROS) production. The fluorescent photomicrograph showed the mitochondrial ROS (red color) in ductus arteriosus smooth muscle cells (DASMCs). Ang II induced ROS production, but when co-treated with cinaciguat, the ROS production declined. The bar graph showed the fluorescence intensity of ROS in different doses. Values represent the mean \pm SEM; $N=6$. One-way ANOVA $P<0.0001$. ${ }^{\# \# P}<0.01$ vs. control; ${ }^{\star \star} P<0.01$ vs. Ang II alone (magnification $200 \times$ ).

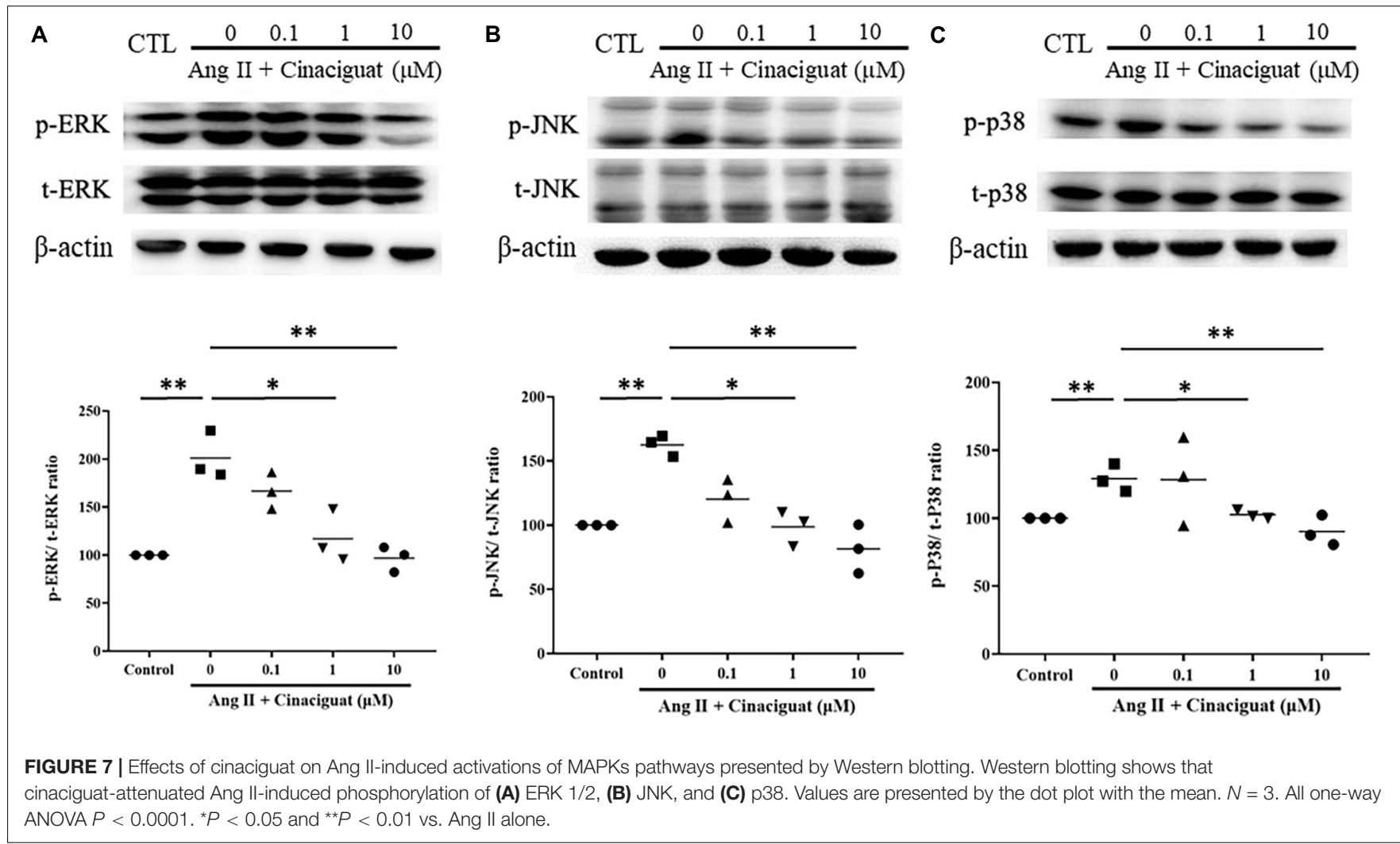




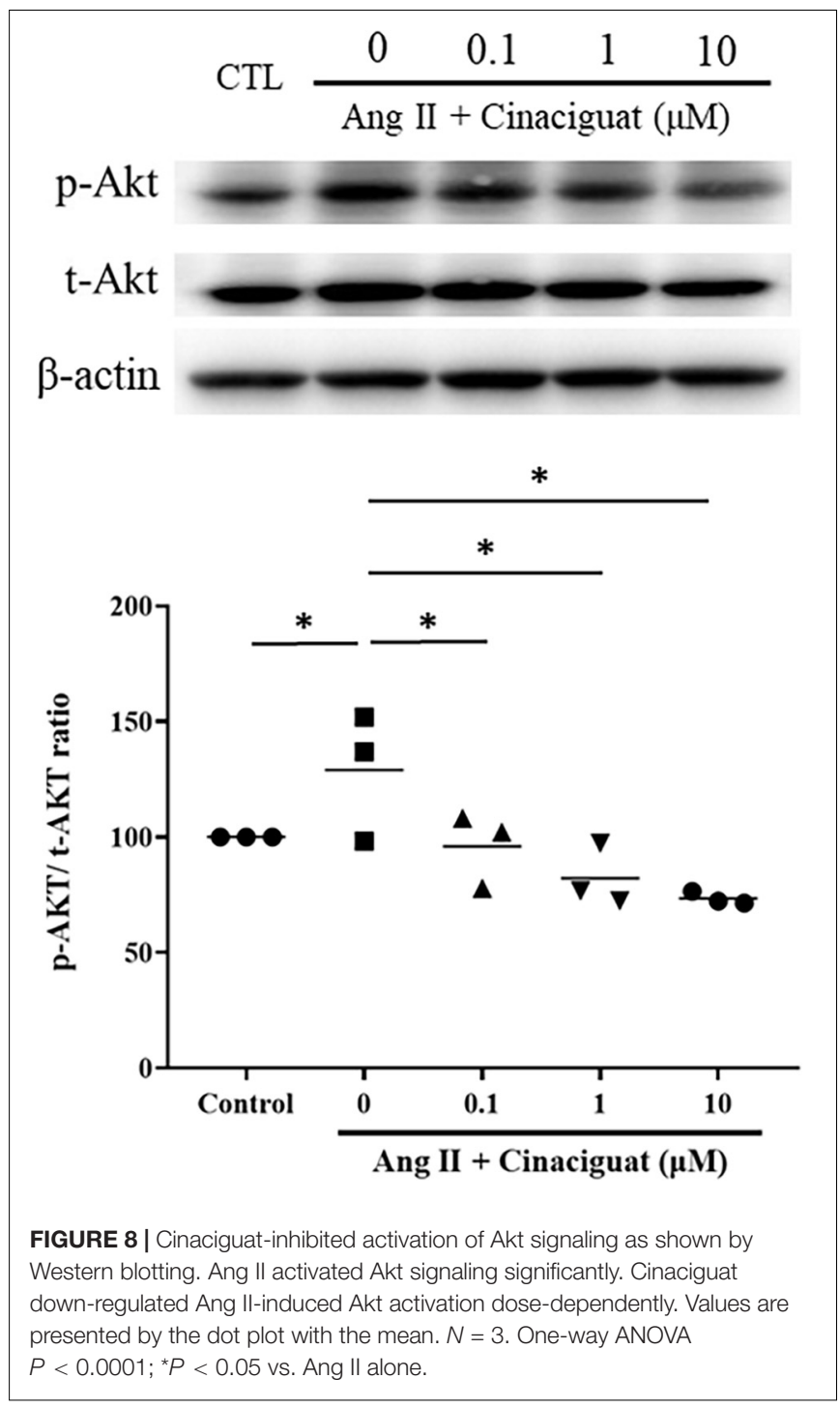

critical for SMC proliferation and migration (Fulton et al., 2017). Furthermore, mitochondria are one of the major sources of ROS (Turrens, 2003), so we examined mitochondrial ROS of DASMCs treated with cinaciguat to study the mechanism underlying the anti-proliferative and ant-migratory effects of cinaciguat. The results demonstrated that cinaciguat attenuated mitochondrial ROS production induced by Ang II. It is known that NADPH oxidase is a classical target of Ang II. However, emerging studies have shown that mitochondria, with crosstalk to NADPH oxidase, is also an essential source of Ang II-mediated ROS in VSMCs (Tsai et al., 2016; Fukai and Ushio-Fukai, 2020). In addition, mitochondria-derived ROS has been recently demonstrated with a key role during DA constriction (Michelakis et al., 2002; Turrens, 2003; Hong et al., 2014). Therefore, instead of NADPH oxidase, in this study, we investigated mitochondria ROS and revealed its role in DASMCs.

The final study was to examine the possible roles of signal transduction involved with anti-proliferative and anti-migratory effects of cinaciguat. It is known that the MAPK family and Akt signaling are both down-stream mediators of ROS, participating in SMC proliferation and migration (Muslin, 2008; Abeyrathna and $\mathrm{Su}, 2015)$. In addition, activation of the cGMP pathway inhibited MAPK and Akt signaling (Bouallegue et al., 2007; Wu et al., 2016). In this study, we found that cinaciguat inhibited Ang II-induced phosphorylation of the MAPK family and Akt dose-dependently, which was possibly because of its effect of enhancing cGMP signaling.

There are some limitations of this study. We did not examine if inhibitors of ERK, JNK, p38, and Akt could attenuate the effects of cinaciguat on DA regulation. In addition, we did not investigate the role of NADPH oxidase, which is a classical target of Ang II and a major source of ROS in VSMC. Thus, these important issues warrant further investigations.

In conclusion, cinaciguat prevents postnatal DA closure by vasodilation and anti-remodeling through the cGMP/PKG pathway. The mechanisms underlying anti-remodeling effects include anti-proliferation and anti-migration, with attenuation of mitochondrial ROS production, MAPKs, and Akt signaling. Thus, this study implicates that sGC activation may be a promising novel strategy to regulate DA patency.

\section{DATA AVAILABILITY STATEMENT}

The original contributions presented in the study are included in the article/Supplementary Material, further inquiries can be directed to the corresponding author/s.

\section{ETHICS STATEMENT}

The animal study was reviewed and approved by Animal Care and Use Committee of the Kaohsiung Medical University. The Institutional Animal Care and Use Committee (IACUC) number is 102094. Animals were cared for in compliance with the guiding principles of the National Institutes of Health of the United States.

\section{AUTHOR CONTRIBUTIONS}

Y-CH wrote the manuscript. J-LY initiated the constructs and validated them, and supervised the experiments. Y-CL and B-NW designed the myograph experiments. J-HH designed the experiments and revised the manuscript. All authors have read and agreed to the published version of the manuscript.

\section{FUNDING}

This study was supported in part by research grants from the Ministry of Science and Technology (MOST 103-2314-B-037040-MY3 and MOST 108-2314-B-037-072-MY3), Kaohsiung Medical University Hospital (KMUH 107-7R45 and KMUH 1088R48), and the Chi-Mei Medical Center and Kaohsiung Medical University Research Foundation, Taiwan (107-CM-KMU-01). 


\section{REFERENCES}

Abeyrathna, P., and Su, Y. (2015). The critical role of Akt in cardiovascular function. Vascul. Pharmacol. 74, 38-48. doi: 10.1016/j.vph.2015. 05.008

Bokenkamp, R., DeRuiter, M. C., van Munsteren, C., and Gittenberger-de Groot, A. C. (2010). Insights into the pathogenesis and genetic background of patency of the ductus arteriosus. Neonatology 98, 6-17. doi: 10.1159/00026 2481

Bouallegue, A., Daou, G. B., and Srivastava, A. K. (2007). Nitric oxide attenuates endothelin-1-induced activation of ERK1/2, PKB, and Pyk2 in vascular smooth muscle cells by a cGMP-dependent pathway. Am. J. Physiol. Heart Circ. Physiol. 293, H2072-H2079. doi: 10.1152/ajpheart.01097. 2006

Chester, M., Seedorf, G., Tourneux, P., Gien, J., Tseng, N., Grover, T., et al. (2011). Cinaciguat, a soluble guanylate cyclase activator, augments cGMP after oxidative stress and causes pulmonary vasodilation in neonatal pulmonary hypertension. Am. J. Physiol. Lung. Cell. Mol. Physiol. 301, L755-L764. doi: 10.1152/ajplung.00138.2010

Clyman, R. I., Waleh, N., Black, S. M., Riemer, R. K., Mauray, F., and Chen, Y. Q. (1998). Regulation of ductus arteriosus patency by nitric oxide in fetal lambs: the role of gestation, oxygen tension, and vasa vasorum. Pediatr. Res. 43, 633-644. doi: 10.1203/00006450-199805000-00012

Crockett, S. L., Berger, C. D., Shelton, E. L., and Reese, J. (2019). Molecular and mechanical factors contributing to ductus arteriosus patency and closure. Congenit. Heart Dis. 14, 15-20. doi: 10.1111/chd. 12714

Dumitrascu, R., Weissmann, N., Ghofrani, H. A., Dony, E., Beuerlein, K., Schmidt, H., et al. (2006). Activation of soluble guanylate cyclase reverses experimental pulmonary hypertension and vascular remodeling. Circulation 113, 286-295. doi: 10.1161/CIRCULATIONAHA.105.581405

Evgenov, O. V., Kohane, D. S., Bloch, K. D., Stasch, J. P., Volpato, G. P., Bellas, E., et al. (2007). Inhaled agonists of soluble guanylate cyclase induce selective pulmonary vasodilation. Am. J. Respir. Crit. Care Med. 176, 1138-1145. doi: 10.1164/rccm.200707-11210C

Evgenov, O. V., Pacher, P., Schmidt, P. M., Hasko, G., Schmidt, H. H., and Stasch, J. P. (2006). NO-independent stimulators and activators of soluble guanylate cyclase: discovery and therapeutic potential. Nat. Rev. Drug Discov. 5, 755-768. doi: $10.1038 / \mathrm{nrd} 2038$

Fukai, T., and Ushio-Fukai, M. (2020). Cross-Talk between NADPH Oxidase and Mitochondria: role in ROS Signaling and Angiogenesis. Cells 9:1849. doi: 10. 3390/cells9081849

Fulton, D. J. R., Li, X., Bordan, Z., Haigh, S., Bentley, A., Chen, F., et al. (2017). Reactive Oxygen and Nitrogen Species in the Development of Pulmonary Hypertension. Antioxidants 6:54. doi: 10.3390/antiox60 30054

Goyal, R., Goyal, D., Longo, L. D., and Clyman, R. I. (2016). Microarray gene expression analysis in ovine ductus arteriosus during fetal development and birth transition. Pediatr. Res. 80, 610-618. doi: 10.1038/pr. 2016.123

Hamrick, S. E., and Hansmann, G. (2010). Patent ductus arteriosus of the preterm infant. Pediatrics 125, 1020-1030. doi: 10.1542/peds.2009-3506

Hamrick, S. E. G., Sallmon, H., Rose, A. T., Porras, D., Shelton, E. L., Reese, J., et al. (2020). Patent Ductus Arteriosus of the Preterm Infant. Pediatrics 146:e20201209. doi: 10.1542/peds.2020-1209

Hirschberg, K., Tarcea, V., Pali, S., Barnucz, E., Gwanmesia, P. N., Korkmaz, S., et al. (2013). Cinaciguat prevents neointima formation after arterial injury by decreasing vascular smooth muscle cell migration and proliferation. Int. J. Cardiol. 167, 470-477. doi: 10.1016/j.ijcard.2012. 01.032

Hong, Z., Cabrera, J. A., Mahapatra, S., Kutty, S., Weir, E. K., and Archer, S. L. (2014). Activation of the EGFR/p38/JNK pathway by mitochondrialderived hydrogen peroxide contributes to oxygen-induced contraction of ductus arteriosus. J. Mol. Med. 92, 995-1007. doi: 10.1007/s00109-0141162-1

Hsu, H. W., Lin, T. Y., Liu, Y. C., Yeh, J. L., and Hsu, J. H. (2021). Molecular Mechanisms Underlying Remodeling of Ductus Arteriosus: looking beyond the Prostaglandin Pathway. Int. J. Mol. Sci. 22:3238. doi: 10.3390/ijms2206 3238

Hsu, J. H., Liou, S. F., Yang, S. N., Wu, B. N., Dai, Z. K., Chen, I. J., et al. (2014). B-type natriuretic peptide inhibits angiotensin II-induced proliferation and migration of pulmonary arterial smooth muscle cells. Pediatr. Pulmonol. 49, 734-744. doi: 10.1002/ppul.22904

Hsu, J. H., Wu, J. R., Liou, S. F., Chen, H. M., Dai, Z. K., Chen, I. J., et al. (2011). Labedipinedilol-A prevents lysophosphatidylcholine-induced vascular smooth muscle cell death through reducing reactive oxygen species production and anti-apoptosis. Atherosclerosis 217, 379-386. doi: 10.1016/j.atherosclerosis. 2011.04.022

Hsu, J. H., Yang, S. N., Chen, H. L., Tseng, H. I., Dai, Z. K., and Wu, J. R. (2010). B-type natriuretic peptide predicts responses to indomethacin in premature neonates with patent ductus arteriosus. J. Pediatr. 157, 79-84. doi: 10.1016/j. jpeds.2009.12.045

Hung, Y. C., Yeh, J. L., and Hsu, J. H. (2018). Molecular Mechanisms for Regulating Postnatal Ductus Arteriosus Closure. Int. J. Mol. Sci. 19:1861. doi: 10.3390/ ijms19071861

Lakshminrusimha, S., Mathew, B., and Leach, C. L. (2016). Pharmacologic strategies in neonatal pulmonary hypertension other than nitric oxide. Semin. Perinatol. 40, 160-173. doi: 10.1053/j.semperi.2015.12.004

Lewis, A. B., Freed, M. D., Heymann, M. A., Roehl, S. L., and Kensey, R. C. (1981). Side effects of therapy with prostaglandin E1 in infants with critical congenital heart disease. Circulation 64, 893-898. doi: 10.1161/01.cir.64. 5.893

Lin, T. Y., Yeh, J. L., and Hsu, J. H. (2020). Role of Extracellular Matrix in Pathophysiology of Patent Ductus Arteriosus: emphasis on Vascular Remodeling. Int. J. Mol. Sci. 21:4761. doi: 10.3390/ijms211 34761

Liou, S. F., Nguyen, T. T. N., Hsu, J. H., Sulistyowati, E., Huang, S. E., Wu, B. N., et al. (2020). The Preventive Effects of Xanthohumol on Vascular Calcification Induced by Vitamin D3 Plus Nicotine. Antioxidants 9:956. doi: 10.3390/antiox9100956

Liou, S. F., Yeh, J. L., Liang, J. C., Chiu, C. C., Lin, Y. T., and Chen, I. J. (2004). Inhibition of mitogen-mediated proliferation of rat vascular smooth muscle cells by labedipinedilol-A through PKC and ERK 1/2 pathway. J. Cardiovasc. Pharmacol. 44, 539-551. doi: 10.1097/00005344-20041100000005

Michelakis, E. D., Rebeyka, I., Wu, X., Nsair, A., Thebaud, B., Hashimoto, K., et al. (2002). O2 sensing in the human ductus arteriosus: regulation of voltage-gated $\mathrm{K}+$ channels in smooth muscle cells by a mitochondrial redox sensor. Circ. Res. 91, 478-486. doi: 10.1161/01.res.0000035057.63 303.d1

Muslin, A. J. (2008). MAPK signalling in cardiovascular health and disease: molecular mechanisms and therapeutic targets. Clin. Sci. 115, 203-218. doi: 10.1042/CS20070430

Oncel, M. Y., and Erdeve, O. (2015). Safety of therapeutics used in management of patent ductus arteriosus in preterm infants. Curr. Drug Saf. 10, 106-112. doi: $10.2174 / 1574886309999141030142847$

Ovali, F. (2020). Molecular and Mechanical Mechanisms Regulating Ductus Arteriosus Closure in Preterm Infants. Front. Pediatr. 8:516. doi: 10.3389/fped. 2020.00516

Seidner, S. R., Chen, Y. Q., Oprysko, P. R., Mauray, F., Tse, M. M., Lin, E., et al. (2001). Combined prostaglandin and nitric oxide inhibition produces anatomic remodeling and closure of the ductus arteriosus in the premature newborn baboon. Pediatr. Res. 50, 365-373. doi: 10.1203/00006450-20010900000012

Tsai, I. C., Pan, Z. C., Cheng, H. P., Liu, C. H., Lin, B. T., and Jiang, M. J. (2016). Reactive oxygen species derived from NADPH oxidase 1 and mitochondria mediate angiotensin II-induced smooth muscle cell senescence. J. Mol. Cell. Cardiol. 98, 18-27. doi: 10.1016/j.yjmcc.2016.07.001

Turrens, J. F. (2003). Mitochondrial formation of reactive oxygen species. J. Physiol. 552, 335-344. doi: 10.1113/jphysiol.2003.049478

Wu, J. R., Yeh, J. L., Liou, S. F., Dai, Z. K., Wu, B. N., and Hsu, J. H. (2016). Gammasecretase Inhibitor Prevents Proliferation and Migration of Ductus Arteriosus Smooth Muscle Cells through the Notch3-HES1/2/5 Pathway. Int. J. Biol. Sci. 12:1063-1073. doi: 10.7150/ijbs. 16430 
Yeh, J. L., Wu, J. R., Wu, B. N., Yang, S. F., Dai, Z. K., Liou, S. F., et al. (2018). B-type natriuretic peptide prevents postnatal closure of ductus arteriosus by both vasodilation and anti-remodeling in neonatal rats. Clin. Sci. 132, 2045-2058. doi: 10.1042/CS2018 0201

Yokoyama, U., Iwatsubo, K., Umemura, M., Fujita, T., and Ishikawa, Y. (2013). The prostanoid EP4 receptor and its signaling pathway. Pharmacol. Rev. 65, 1010-1052. doi: 10.1124/pr.112.007195

Conflict of Interest: The authors declare that the research was conducted in the absence of any commercial or financial relationships that could be construed as a potential conflict of interest.
Publisher's Note: All claims expressed in this article are solely those of the authors and do not necessarily represent those of their affiliated organizations, or those of the publisher, the editors and the reviewers. Any product that may be evaluated in this article, or claim that may be made by its manufacturer, is not guaranteed or endorsed by the publisher.

Copyright (๑) 2021 Hung, Liu, Wu, Yeh and Hsu. This is an open-access article distributed under the terms of the Creative Commons Attribution License (CC BY). The use, distribution or reproduction in other forums is permitted, provided the original author(s) and the copyright owner(s) are credited and that the original publication in this journal is cited, in accordance with accepted academic practice. No use, distribution or reproduction is permitted which does not comply with these terms. 